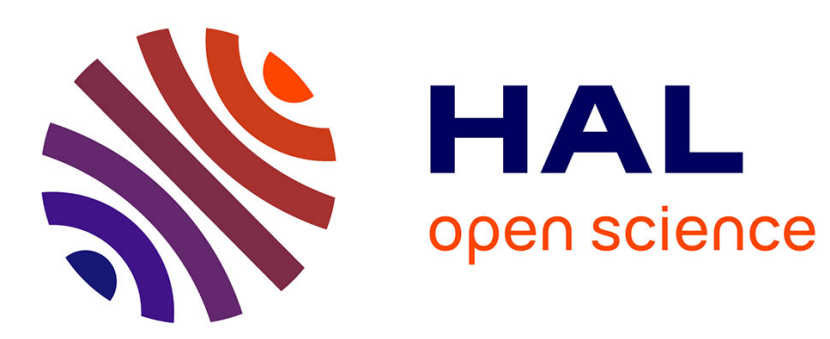

\title{
Interface domain wall and exchange bias phenomena in ferrimagnetic/ferrimagnetic bilayers
}

\author{
S. Mangin, F. Montaigne, A. Schuhl
}

\section{To cite this version:}

S. Mangin, F. Montaigne, A. Schuhl. Interface domain wall and exchange bias phenomena in ferrimagnetic/ferrimagnetic bilayers. Physical Review B: Condensed Matter and Materials Physics (19982015), 2003, 68 (14), 10.1103/PhysRevB.68.140404 . hal-02104715

\section{HAL Id: hal-02104715 \\ https://hal.univ-lorraine.fr/hal-02104715}

Submitted on 19 Apr 2019

HAL is a multi-disciplinary open access archive for the deposit and dissemination of scientific research documents, whether they are published or not. The documents may come from teaching and research institutions in France or abroad, or from public or private research centers.
L'archive ouverte pluridisciplinaire HAL, est destinée au dépôt et à la diffusion de documents scientifiques de niveau recherche, publiés ou non, émanant des établissements d'enseignement et de recherche français ou étrangers, des laboratoires publics ou privés. 


\title{
Interface domain wall and exchange bias phenomena in ferrimagnetic/ferrimagnetic bilayers
}

\author{
S. Mangin, F. Montaigne, and A. Schuhl \\ Laboratoire de Physique des Matériaux, Université Nancy 1, Boîte Postale 239, F-54506 Vandoeuvre lès Nancy, France
}

(Received 12 May 2003; published 3 October 2003)

\begin{abstract}
In $\mathrm{Gd}_{40} \mathrm{Fe}_{60} / \mathrm{Tb}_{12} \mathrm{Fe}_{88}$ exchange-coupled bilayer system, both negative and positive exchange bias, depending on cooling field, are observed. A full enlightenment of the magnetic configurations adopted by the system is obtained through micromagnetic calculations in agreement with magnetization and susceptibility measurements. Magnetization shifts and exchange bias fields are then quantitatively correlated to the presence of a frozen interface domain wall. This model may be transposed to antiferromagnetically exchange coupled antiferromagnetic/ferromagnetic $\left(\mathrm{Fe} / \mathrm{FeF}_{2}\right.$ or $\left.\mathrm{Fe} / \mathrm{MnF}_{2}\right)$ systems
\end{abstract}

DOI: 10.1103/PhysRevB.68.140404

PACS number(s): 75.60.Ch, 75.30.Et, 75.50.Gg, 75.70.Cn

Exchange bias (EB) has been widely studied in ferromagnetic (FM)/antiferromagnetic (AFM) bilayers. ${ }^{1,2}$ This phenomenon which is often essential for magnetoelectronic devices ${ }^{3}$ is mainly characterized, below a blocking temperature $T_{B}$, by a shift of the $M-H$ loop towards a field known as the exchange bias field $H_{E}$. In some FM/AFM systems exhibiting antiferromagnetic exchange coupling such as $\mathrm{Fe} / \mathrm{FeF}_{2}$ or $\mathrm{Fe} / \mathrm{MnF}_{2}$, it has been shown that a cooling field $H_{f c}$ could trigger $H_{E}$ from positive to negative values at low temperature and induce a magnetization shift. ${ }^{4-6}$ Also strong correlation between $H_{f c}, H_{E}$ and magnetization shift of the hysteresis loop has been observed. ${ }^{5}$ Additionally several authors $^{6,7}$ pointed a peak in the coercive field $H_{C}$ for $H_{E}$ close to zero. Despite numerous studies, the mechanism leading to the EB effect is not yet fully understood. However, an estimation of the $H_{E}$ must rely on configurations of the interface magnetization. Two different kinds of model are proposed. A first approach to model EB phenomena is based on the formation of lateral domains in the antiferromagnet resulting in the formation of lateral domain wall. ${ }^{8}$ On the other hand, various models proposed the presence of an interface domain wall (iDW) with a modulation vector perpendicular to the interface. The position of the iDW (in AFM or in the FM) and its shape $\left(90^{\circ}\right.$ or $\left.180^{\circ}\right)$ are up to now widely discussed. ${ }^{9-11}$ These aspects are still in debate after 15 years of intensive research, mainly because the magnetic configuration inside the AFM layer is not measurable using classical magnetometric methods (at least linear) due to its zero net magnetization. Other exchange-coupled systems such as "spring magnet" made of two layers (ferromagnetic or/and ferrimagnetic) have shown EB effect. ${ }^{12-14}$ The presence of an interface DW was clearly evidence in that case. However, the interface exchange coupling was ferromagnetic and only negative EB was observed.

In this paper, we have studied EB phenomena in an antiferromagnetically coupled ferrimagnetic/ferrimagnetic bilayer, namely, $\mathrm{Gd}_{40} \mathrm{Fe}_{60} / \mathrm{Tb}_{12} \mathrm{Fe}_{88}$. We present here the evolution of the magnetic properties $\left(H_{E}, H_{C}\right.$, and magnetization shifts) of this system at low temperature depending on $H_{f c}$. Using ferrimagnetic layers, we investigated the magnetic configurations of both layers, and then develop a quantitative model for $H_{E}$ and $M_{\text {Shift }}^{N}$ variation of antiferromagnetically coupled bilayers. The $\mathrm{Gd}_{40} \mathrm{Fe}_{60}(100 \mathrm{~nm}) / \mathrm{Tb}_{12} \mathrm{Fe}_{88}(50 \mathrm{~nm})$ sample has been prepared by coevaporation on a glass substrate kept at $77 \mathrm{~K}$ and protected by a 30-nm Si capping layer. ${ }^{12}$ Both $\mathrm{Gd}_{40} \mathrm{Fe}_{60}$ and $\mathrm{Tb}_{12} \mathrm{Fe}_{88}$ are ferrimagnetic alloys, $\mathrm{Fe}$ and rare-earth magnetic moments being very strongly antiferromagnetically coupled and thus antiparallel. In $\mathrm{Tb}_{12} \mathrm{Fe}_{88}$, the iron contribution to magnetization is dominant for all temperatures. This composition has been chosen to avoid the occurrence of a compensation temperature. ${ }^{16}$ The terbium moments present a spreading of their direction because of the strong random local anisotropy. $\mathrm{Tb}_{12} \mathrm{Fe}_{88}$ becomes thus a hard magnetic alloy at low temperature because of the large increase of the terbium magnetic anisotropy. On the contrary, in $\mathrm{Gd}_{40} \mathrm{Fe}_{60}$, the contribution of gadolinium is dominant and the magnetization is locally parallel to that of the Gd moments (antiparallel to that of iron), the magnetic anisotropy is generated by the growth process and $\mathrm{GdFe}$ exhibits a very clear in-plane easy axis in the film plane. ${ }^{17}$ This anisotropy remains weak at low temperatures because of the $s$ character of the Gd $4 f$ band. For all the measurements presented here, the magnetic field was applied in the sample plane along the GdFe easy axis. The exchange coupling between the layers being dominated by $\mathrm{Fe}-\mathrm{Fe}$ ferromagnetic interactions, the magnetizations of $\mathrm{GdFe}$ and $\mathrm{TbFe}$ are antiferromagnetically coupled at the interface.

Equilibrium magnetic configurations have been calculated by using a simple unidimensional micromagnetic model. ${ }^{13,14}$ Due to the demagnetizing field, the magnetization is kept in the plane of the layers and the magnetic profile is characterized by a single depth dependent angle $\theta(z)$ referred to the anisotropy direction. The magnetic energy of the bilayer per area unit is calculated by considering, for each layer $(\mathrm{GdFe}$ and $\mathrm{TbFe}$ ): the exchange energy (characterized by the exchange stiffness $A_{\mathrm{GdFe}}$ or $A_{\mathrm{TbFe}}$ ), the anisotropy energy (minimum for $\theta=0^{\circ}$ and $\theta=180^{\circ}$ and proportional to the anisotropy energy $K_{\mathrm{GdFe}}$ or $K_{\mathrm{TbFe}}$ ), the Zeeman energy corresponding to the interaction of the magnetization $M_{\mathrm{GdFe}}$ or $M_{\mathrm{TbFe}}$ with the applied field $H$, and finally the interface exchange energy (characterized by a negative exchange stiffness $J$ ). 
TABLE I. Magnetic parameters of a single layer (thickness $t$ ) $\mathrm{Gd}_{40} \mathrm{Fe}_{60}$ and $\mathrm{Tb}_{12} \mathrm{Fe}_{88}$ at $300 \mathrm{~K}$ and $5 \mathrm{~K}$ used to performed the micromagnetic simulations on the bilayer. (The interface coupling $J$ has been taken to be $-10^{-7} \mathrm{erg} / \mathrm{cm}^{2}$ ). They are found to be in good agreement with the one obtained from measurements and calculations (Ref. 16).

\begin{tabular}{lcccc}
\hline \hline Alloy & $t(\mathrm{~nm})$ & $A(\mathrm{erg} / \mathrm{cm})$ & $K\left(\mathrm{erg} / \mathrm{cm}^{3}\right)$ & $M(\mathrm{emu})$ \\
\hline $\mathrm{Gd}_{40} \mathrm{Fe}_{60}(300 \mathrm{~K})$ & 100 & $6 \times 10^{-7}$ & $2.5 \times 10^{4}$ & 500 \\
$\mathrm{Gd}_{40} \mathrm{Fe}_{60}(5 \mathrm{~K})$ & 100 & $6 \times 10^{-7}$ & $10^{5}$ & 1000 \\
$\mathrm{~Tb}_{12} \mathrm{Fe}_{88}(300 \mathrm{~K})$ & 50 & $8 \times 10^{-8}$ & $2.8 \times 10^{4}$ & 225 \\
$\mathrm{~Tb}_{12} \mathrm{Fe}_{88}(5 \mathrm{~K})$ & 50 & $8 \times 10^{-8}$ & $>10^{7}$ & 350 \\
\hline \hline
\end{tabular}

$$
\begin{aligned}
E= & \int_{0}^{t_{\mathrm{GdFe}}}\left[A_{\mathrm{GdFe}}\left(\frac{\partial \theta(z)}{\partial z}\right)^{2}+K_{\mathrm{GdFe}} \sin ^{2} \theta(z)\right. \\
& \left.-H M_{\mathrm{GdFe}} \cos \theta(z)\right] d z+\int_{t_{\mathrm{GdFe}}}^{t_{\mathrm{GdFe}}+t_{\mathrm{TbFe}}}\left[A_{\mathrm{TbFe}}\left(\frac{\partial \theta(z)}{\partial z}\right)^{2}\right. \\
& \left.+K_{\mathrm{TbFe}} \sin ^{2} \theta(z)-H M_{\mathrm{TbFe}} \cos \theta(z)\right] d z \\
& +J \cos \left(\theta_{\mathrm{TbFe}}^{i}-\theta_{\mathrm{GdFe}}^{i}\right)
\end{aligned}
$$

where $\theta_{\mathrm{GdFe}}^{i}$ and $\theta_{\mathrm{TbFe}}^{i}$ are, respectively, the angle of the magnetization at the interface in $\mathrm{GdFe}$ and in the TbFe layer. We determined the magnetic profiles which minimize the energy (stable and metastables states) from which we calculated the magnetization $M$ and the susceptibility $\partial M / \partial H$. The magnetic parameters used for the calculation are summarized in Table I.

Magnetic measurements were first performed at $300 \mathrm{~K}$ to establish the possible initial magnetic configurations before cooling the sample under a magnetic field $H_{f c}$. Experimental results are reported in Fig. 1 for both magnetization and susceptibility. The hysteresis loops as a function of the applied magnetic field are plotted along with the results of the micromagnetic calculations. For the clarity of the figure, only the absolute minimum-energy solution is represented. The agreement between measurement and micromagnetic calculations is then very good for both magnetization and susceptibility. The solutions corresponding to local minima (not represented in Fig. 1) reproduce satisfactorily the opening of the loops. The magnetic profile $\theta(z)$ from the calculation for five different fields are presented in inset in Fig. 2. For very large positive fields $(H=70 \mathrm{kOe})$, both magnetizations point in the field direction with only a small shift at the interface, consequently the angle between the interface $\mathrm{GdFe}$ and $\mathrm{TbFe}$ magnetizations and the field direction are close to zero ( $\theta_{\mathrm{GdFe}}^{i} \simeq 0^{\circ}$ and $\theta_{\mathrm{TbFe}}^{i} \simeq 0^{\circ}$ ). As the field is decreased the balance between Zeeman energy and the interfacial antiferromagnetic coupling leads to the formation of an iDW, mainly located in the TbFe layer (inset of Fig. 2). Its thickness increases and $\theta_{\mathrm{TbFe}}^{i}$ (calculated) rises from $0^{\circ}$ angle toward $180^{\circ}$ (Fig. 2). This "decompression" of the iDW is slightly visible from the slow decrease of the magnetization, but it is clearly evidenced by the susceptibility measurements. Indeed, when a DW becomes larger, the amount of

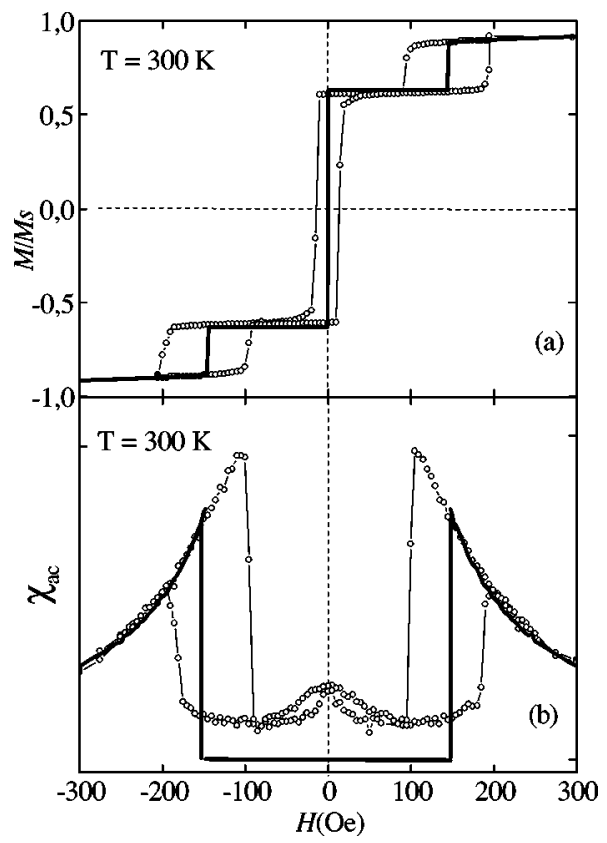

FIG. 1. Normalized magnetization $M / M_{S}$ (a) and susceptibility $\chi_{\text {ac }}($ b) as a function of the magnetic field $H$ applied along the easy axis for $\mathrm{Gd}_{40} \mathrm{Fe}_{60}(100 \mathrm{~nm}) / \mathrm{Tb}_{12} \mathrm{Fe}_{88}(50 \mathrm{~nm})$ at $300 \mathrm{~K}$. Experimental data (open circle) are compared to calculated values (solid line).

magnetization having a component perpendicular to the field direction increases, which increases the susceptibility. ${ }^{12}$ The susceptibility is maximum at $H=100$ Oe field at which the antiferromagnetic coupling takes over the Zeeman energy and the TbFe magnetization reverses abruptly (Fig. 1). The magnetizations are thus antiparallel and entirely along the applied field $\left(\theta_{\mathrm{GdFe}}^{i}=0^{\circ}\right.$ and $\theta_{\mathrm{TbFe}}^{i}=180^{\circ}$ ) without any iDW. This transition leads to a drop of the susceptibility because of the disappearance of transverse magnetization component. For a small negative field, the whole structure (GdFe and TbFe still antiferromagntically aligned) reverses its magnetization (Fig. 2), GdFe magnetization is now pointing in the negative field direction $\left(\theta_{\mathrm{GdFe}}^{i}=180^{\circ}\right.$ and $\left.\theta_{\mathrm{TbFe}}^{i}=0^{\circ}\right)$. For $H<-200 \mathrm{Oe}$, Zeeman energy takes over interfacial cou-

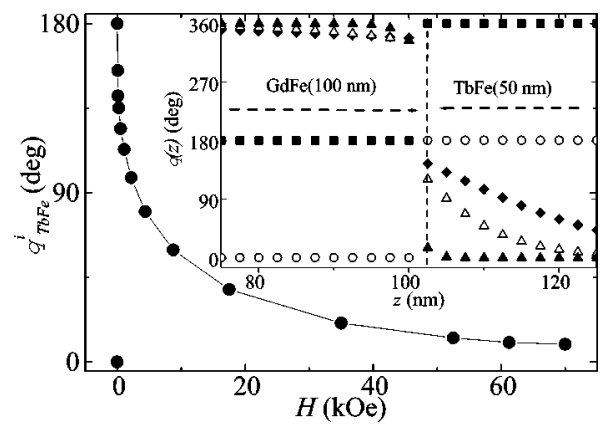

FIG. 2. Calculated angle $\theta_{\mathrm{TbFe}}^{i}$ between the TbFe interface spin and the cooling field direction as a function of the applied field $H$. In inset, magnetic configurations in the $\mathrm{GdFe} / \mathrm{TbFe}$ bilayer at 300 $\mathrm{K}, \theta(z)$, for various fields $70 \mathrm{kOe}$ (full triangle), $1 \mathrm{kOe}$ (open triangle), 150 Oe (full diamond), and 0 Oe. For the zero field two configurations are possible (open circle) and (full square). 


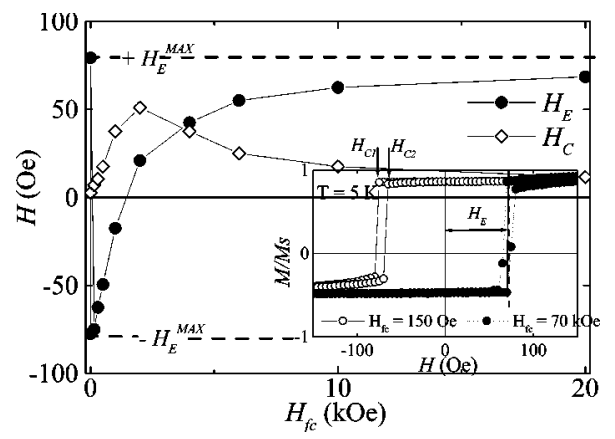

FIG. 3. Variation of the exchange bias field $H_{E}$ and the coercive field $H_{C}$ as a function of the cooling field $H_{f c}$. In inset, hysteresis loops at $5 \mathrm{~K}$ for $H_{f c}=70 \mathrm{kOe}$ and $H_{f c}=150 \mathrm{Oe} . H_{C 1}$ and $H_{C 2}$ are defined for $H_{f c}=150 \mathrm{Oe}$

pling and the TbFe magnetization aligns along the field, leading again to the creation of an iDW. Symmetric behavior is observed while the field is swept back to positive. The roomtemperature behavior of the $\mathrm{GdFe} / \mathrm{TbFe}$ bilayer is similar to the one obtained for $\mathrm{GdFe} / \mathrm{FeSn}$ antiferromagnetically coupled systems. ${ }^{15}$ It is thus perfectly understood and conform to a simple micromagnetic model which allows to predict the magnetic configuration at the TbFe interface, and particularly $\theta_{\mathrm{TbFe}}^{i}$ represented in Fig. 2 .

The sample was then cooled down to $5 \mathrm{~K}$ under constant magnetic fields $H_{f c}$. Two examples of $5 \mathrm{~K}$ hysteresis loop are represented in the inset of Fig. 3. The net bias field appearing in the cycle depends clearly on the cooling field $H_{f c}$. This phenomenon is accompanied by a magnetization shift, whose variation with $H_{f c}$ is reported in Fig. 4(b). The two switching fields $H_{C 1}$ and $H_{C 2}$ are plotted as a function of $H_{f c}$ in Fig. 4(a). As usual, we define $H_{E}=\left(H_{C 2}+H_{C 1}\right) / 2$ and the coercivity as $H_{C}=\left(H_{C 2}-H_{C 1}\right) / 2$, both plotted in Fig. 3 as a function of the cooling field. The exchange field is maximal in negative values $\left(H_{E}=-H_{E}^{\max }\right)$ for $H_{f c}=0$ $\left(\theta_{\mathrm{TbFe}}^{i}=180^{\circ}\right)$ and increases monotonically with $H_{f c}$. It is remarkable that for large values of $H_{f c}\left(\theta_{\mathrm{TbFe}}^{i}=0^{\circ}\right), H_{E}$ tends toward $+H_{E}^{\max }$ (which is also the exchange field for $H_{f c}=0$ with $\left.\theta_{\mathrm{TbFe}}^{i}=0^{\circ}\right) . H_{C}$ is minimum for low and large values of $H_{f c}$ and maximum for an intermediate value corresponding to an $H_{f c}$ for which $H_{E}$ is almost zero. It appears clearly that $H_{E}$ is related to $H_{f c}$ and particularly to the interface magnetic configuration in the TbFe before the cooling. Indeed from $H_{E}=-H_{E}^{\max }$ for $\theta_{\mathrm{TbFe}}^{i}=180^{\circ}, H_{E}$ increases to $+H_{E}^{\max }$ for $\theta_{\mathrm{TbFe}}^{i}=0^{\circ}$ going through $H_{E}=0$ for about $\theta_{\mathrm{TbFe}}^{i}= \pm 90^{\circ}$

To quantify this relationship, we suppose below that the 300-K magnetic configuration inside the TbFe is frozen during the cooling procedure and remained unchanged at $5 \mathrm{~K}$. Consequently, $\theta_{\mathrm{TbFe}}^{i}\left(H=H_{f c}\right)$ at $300 \mathrm{~K}$ is equal to $\theta_{\mathrm{TbFe}}^{i}\left(H_{f c}\right)$ at $5 \mathrm{~K}$. Micromagnetic calculations have been performed for various fixed $\mathrm{TbFe}$ configurations deduced from $300 \mathrm{~K}$ calculations and using GdFe magnetic parameters for $5 \mathrm{~K}$ (Table I) considering that the magnetization of $\mathrm{TbFe}$ is frozen. The field $H_{C 1}^{c a l c}$ for which it is energetically favorable for the $\mathrm{GdFe}$ magnetization to reverse was then deduced and found to be a linear function of $\theta_{\mathrm{TbFe}}^{i}$. The

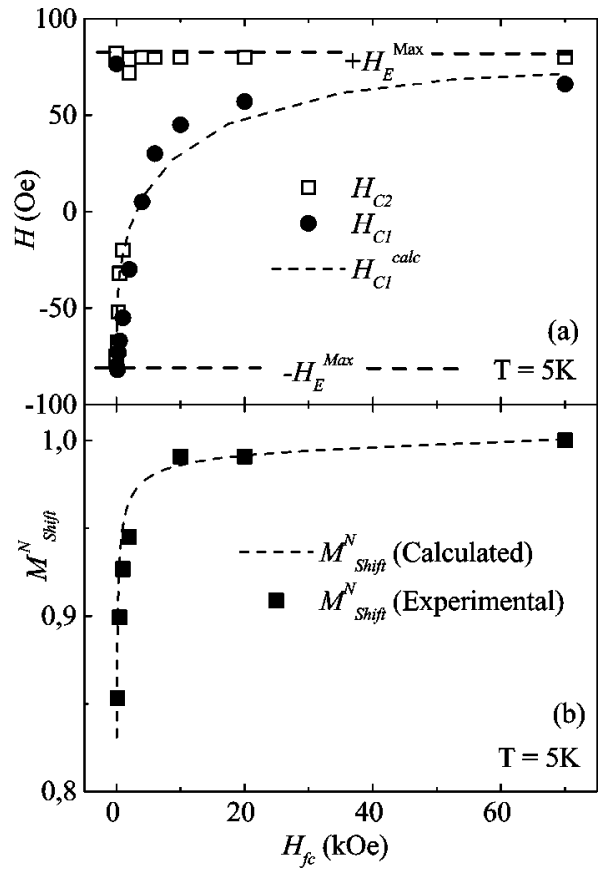

FIG. 4. (a) Evolution of the calculated field $H_{C 1}^{\text {calc }}$ at which GdFe reverses (dash line) compared to $H_{C 1}$ (full circle) and $H_{C 2}$ (open square) as a function of the cooling field $H_{f c}$. (b) Cooling field dependence of the experimental (full square) and calculated (dash line) normalized magnetization shift $M_{\text {Shift }}^{N}\left(H_{f c}\right)$.

$H_{C 1}^{\text {calc }}$ variation as a function of $H_{f c}$ is compared to $H_{C 1}$ variation in Fig. 4(a). The agreement between the experimental results and the frozen magnetic configuration hypothesis is good, showing the relevance of iDW to explain exchange bias phenomena in our system. The difference between $H_{C 2}$ and $H_{C 1}$, the coercivity, is superior to the intrinsic coercivity of $\mathrm{GdFe}$ and upon a certain value of cooling field $\left(H_{f c}\right.$ $>4 \mathrm{kOe}), H_{C 2}$ is constant and equal to $+H_{E}^{\max }$. These points suggest that during the $\mathrm{GdFe}$ reversal at $H_{C 1}$, a rearrangement of the TbFe interface magnetization occurs with a reduction of $\theta_{\mathrm{TbFe}}^{i}$. For $\theta_{\mathrm{TbFe}}^{i}<90^{\circ}$ (corresponding to $H_{f c}$ $>4 \mathrm{kOe}$ ), the rearrangement leads directly to $\theta_{\mathrm{TbFe}}^{i}=0^{\circ}$ and $H_{C 2}=+H_{E}^{\max }$. The amplitude of the rearrangement is directly given by the coercivity (represented in Fig. 3). The amplitude is maximum for low exchange bias fields. The torque "exerted" by the GdFe during its reversal on the TbFe interface is thus maximum for $\theta_{\mathrm{TbFe}}^{i}=90^{\circ}$. Nevertheless, this rearrangement does not limit our quantitative analysis in first stage of the $M-H$ loop.

Let us point out that the results described above for $\mathrm{Gd}_{40} \mathrm{Fe}_{60} / \mathrm{Tb}_{12} \mathrm{Fe}_{88}$ are in all respect similar to previous observations done on $\mathrm{Fe} / \mathrm{FeF}_{2}$ and $\mathrm{Fe} / \mathrm{FeMn}_{2}$ systems. ${ }^{4,5}$ It actually gives credit to new theoretical and experimental development ${ }^{18}$ showing that in the AFM the moments tend to align along the field axis as it is the case for TbFe. Moreover, the magnetization shift [Fig. 4(b)] observed in both systems can be easily explained by our model. Indeed the presence of the interfacial DW changes the net magnetization of the system. We then defined the normalized magnetization shift $M_{\text {Shift }}^{N}\left(H_{f c}\right)$ for a given cooling field $H_{f c}$ as $M_{\text {Shift }}^{N}\left(H_{f c}\right)$ 
$=M_{2 \mathrm{kOe}}\left(H_{f c}\right) / M_{2 \mathrm{kOe}}\left(H_{f c}=70 \mathrm{kOe}\right)$ where $M_{2 \mathrm{kOe}}\left(H_{f c}\right)$ is the magnetization measured at $5 \mathrm{~K}$ under $2 \mathrm{kOe}$ after cooling the sample from $300 \mathrm{~K}$ under $H_{f_{c}}$. The calculated and experimental results are in very good agreement as shown in Fig. 4. Moreover for $\mathrm{Fe} / \mathrm{FeF}_{2}$ and $\mathrm{Fe} / \mathrm{FeMn}_{2}$, the $H_{f_{c}}$ dependence of the magnetization shift is qualitatively the same. However, because the net magnetization of AFM is zero, this shift is about $1 \%$ of the total magnetization whereas in our case it reaches $10 \%$.

In conclusion, by using a ferrimagnetic/ferrimagnetic bilayer, we have investigated the magnetic configurations inside an exchange-coupled bilayer system at a temperature $(\mathrm{T}=300 \mathrm{~K})$ above the blocking temperature for which exchange bias appears. The cooling field dependence of the exchange bias phenomena $\left(H_{E}, H_{C}, M_{\text {Shift }}^{N}\right)$ were then explained considering laterally uniform magnetization and the freezing of the magnetic configuration in the pinning layer $(\mathrm{TbFe})$. However no lateral domain formation in either of the two layer is required. More precisely the exchange bias field value is dominated by the angle between the magnetization of the interface pinned layer and the cooling field direction, whereas the magnetization shift is related to the interface magnetic configuration. The model developed here for antiferromagnetically coupled bilayers may be transposed from ferrimagnetic/ferrimagnetic to FM/AFM systems which explains all the reported experimental results for $\mathrm{Fe} / \mathrm{FeF}_{2}$ and $\mathrm{Fe} / \mathrm{MnF}_{2}$ systems and gives credit to interface domain-wall models. ${ }^{9-11}$

We thank M. Hehn and Y. Henry for interesting and fruitful discussions, L. Joly for his help in the micromagnetic calculations, and M. Alnot, F. Mouginet, and D. Pierre for help with experiments.
${ }^{1}$ W.H. Meiklejohn and C.P. Bean, Phys. Rev. 105, 904 (1957).

${ }^{2}$ A.E. Berkowitz and K. Takano, J. Magn. Magn. Mater. 200, 552 (1999); J. Nogues and I.K. Schuller, ibid. 192, 203 (1999); M. Kiwi, ibid. 234, 584 (2001).

${ }^{3}$ J.R. Childress et al., IEEE Trans. Magn. 37, 1745 (2001).

${ }^{4}$ J. Nogues, D. Lederman, T.J. Moran, and Ivan K. Schuller, Phys. Rev. Lett. 76, 4624 (1996).

${ }^{5}$ J. Nogues, C. Leighton, and I.K. Schuller, Phys. Rev. B 61, 1315 (2000).

${ }^{6}$ C. Leighton, M.R. Fitzsimmons, A. Hoffmann, J. Dura, C.F. Majkrzak, M.S. Lund, and I.K. Schuller, Phys. Rev. B 65, 064403 (2002); C. Leighton, J. Nogues, B.J. Josson-Akerman, and I.K. Schuller, Phys. Rev. Lett. 84, 3466 (2000).

${ }^{7}$ T.L. Kirk, O. Hellwig, and E.E. Fullerton, Phys. Rev. B 65, 224426 (2002).

${ }^{8}$ A.P. Malozemoff, J. Appl. Phys. 63, 3874 (1988).

${ }^{9}$ D. Mauri, H.C. Siegmann, P.S. Bagus, and E. Kay, J. Appl. Phys. 62, 3047 (1987).
${ }^{10}$ M. Kiwi, J. Mejia-Lopez, R.D. Portugal, and R. Ramirez, Europhys. Lett. 48, 573 (1999).

${ }^{11}$ R.L. Stamps, J. Magn. Magn. Mater. 242-245, 139 (2002).

${ }^{12}$ S. Mangin, G. Marchal, and B. Barbara, Phys. Rev. Lett. 82, 4336 (1999).

${ }^{13}$ E.E. Fullerton, J.S. Jiang, M. Grimsditch, C.H. Sowers, and S.D. Bader, Phys. Rev. B 58, 12193 (1998).

${ }^{14}$ F. Montaigne, S. Mangin, and Y. Henry, Phys. Rev. B 67, 144412 (2003).

${ }^{15}$ F. Canet, S. Mangin, C. Bellouard, and M. Piecuch, Europhys. Lett. 52, 594 (2000).

${ }^{16}$ P. Hansen, C. Clausen, G. Much, M. Rosenkranz, and K. Witter, J. Appl. Phys. 66, 756 (1989).

${ }^{17}$ S. Mangin, C. Bellouard, G. Marchal, and B. Barbara, J. Magn. Magn. Mater. 165, 161 (1997).

${ }^{18}$ U. Nowak, K.D. Usadel, J. Keller, P. Miltnyi, B. Beschoten, and G. Guntherodt, Phys. Rev. B 67, 014430 (2002). 